\title{
Paraneoplastic motor neuronopathy and malignant acanthosis nigricans
}

\author{
Neuronopatia motora paraneoplásica e acantose nigricans maligna
}

Wladimir Bocca Vieira de Rezende PINTO', Bruno de Mattos Lombardi BADIA', Luiz Henrique Libardi SILVA', Igor Braga FARIAS', Paulo Victor Sgobbi de SOUZA', Acary Souza Bulle OLIVEIRA

A 40-year-old woman presented with rapidly progressive quadriparesis and severe weight loss. Examination disclosed global amyotrophy, absent deep tendon reflexes and malignant acanthosis nigricans (Figure). Neurophysiologic studies disclosed pure motor neuronopathy. Paraneoplastic screening with fullbody CT/MRI scan revealed an intravesical mural lesion (Figure) and urethrocystoscopy showed low-grade papillary urothelial carcinoma of the urinary bladder. High-titer serum anti-Hu antibodies were identified. There was marked motor and dermatological improvement after lesion removal, combined with intravenous immunoglobulin and methylprednisolone.

Dermatological signs are key diagnostic clues in the suspicion of underlying malignancies ${ }^{1}$, including rarely urological malignancies $^{2}$, in the context of paraneoplastic motor neuronopathy ${ }^{3,4}$.

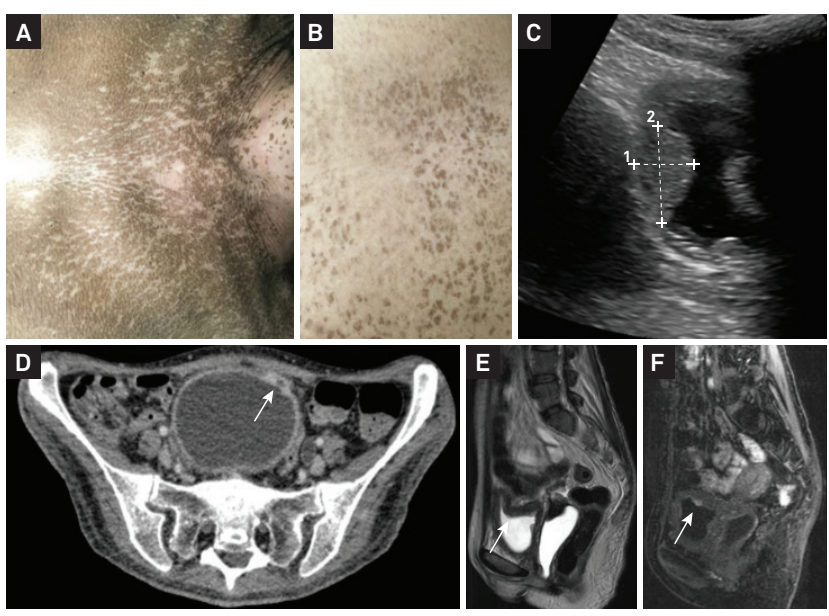

Figure. Malignant acanthosis nigricans and paraneoplastic screening. (A,B) Symmetric hyperpigmented cutaneous lesions without mucosal involvement. Paraneoplastic imaging screening disclosing an intramural intravesical lesion (white arrow) in ultrasonography study (C), abdominal and pelvic CT (D) and T2-weighted (E) and post-gadolinium STIR (F) sequences in MR imaging of the abdomen and pelvis.

\section{References}

1. Silva JA, Mesquita KC, Igreja AC, Lucas IC, Freitas AF,

Oliveira SM, et al. Paraneoplastic cutaneous manifestations: concepts and updates. An Bras Dermatol. 2013 Jan-Feb;88(1):9-22. https://doi.org/10.1590/S0365-05962013000100001

2. Sacco E, Pinto F, Sasso F, Racioppi M, Gulino G, Volpe A, et al. Paraneoplastic syndromes in patients with urological malignancies. Urol Int. 2009;83(1):1-11. https://doi.org/10.1159/000224860
3. Sharp L, Vernino S. Paraneoplastic neuromuscular disorders. Muscle Nerve. 2012 Dec;46(6):841-50. https://doi.org/10.1002/mus.23502

4. Mélé N, Berzero G, Maisonobe T, Salachas F, Nicolas G, Weiss N, et al. Motor neuron disease of paraneoplastic origin: a rare but treatable condition. J Neurol. 2018 Jul;265(7):1590-9. https://doi.org/10.1007/s00415-018-8881-0

\footnotetext{
${ }^{1}$ Universidade Federal de São Paulo; Departamento de Neurologia e Neurocirurgia, São Paulo SP, Brasil.

Paulo Victor Sgobbi de Souza iD https://orcid.org/0000-0002-7416-7108; Bruno de Mattos Lombardi Badia iD https://orcid.org/0000-0001-7360-0494; Wladimir Bocca Vieira de Rezende Pinto (iD) https://orcid.org/0000-0002-0150-525X;Acary Souza Bulle Oliveira iD https://orcid.org/0000-0002-6986-4937 Correspondence: Wladimir Bocca Vieira de Rezende Pinto; Departamento de Neurologia e Neurocirurgia / UNIFESP; Rua Estado de Israel, 899; 04022-002 São Paulo SP, Brasil; E-mail:wladimirbvrpinto@gmail.com

Ethical statement: Full consent was obtained from the patient for the case report. This study was approved by our Ethics Institution.

Conflict of interest: There is no conflict of interest to declare.
}

Received 22 December 2018; Accepted 11 March 2019. 